Bull. Austral. Math. Soc.

$35 \times 55,35 \mathrm{~K} 50,35 \mathrm{R} 70$

Vol. 51 (1995) [501-509]

\title{
IMPLICIT PARABOLIC DIFFERENTIAL EQUATIONS
}

\section{Salvatore Bonafede and Salvatore A. Marano}

Let $Q_{T}=\Omega \times(0, T)$, where $\Omega$ is a bounded domain in $\mathbf{R}^{n}(n \geqslant 3)$ having the cone property and $T$ is a positive real number; let $Y$ be a nonempty, closed connected and locally connected subset of $\mathbf{R}^{h}$; let $f$ be a real-valued function defined in $Q_{T} \times \mathbf{R}^{h} \times \mathbf{R}^{n h} \times Y$; let $\mathcal{L}$ be a linear, second order, parabolic operator. In this paper we establish the existence of strong solutions $u \in W_{p}^{2,1}\left(Q_{T}, \mathbb{R}^{h}\right)$ $(n+2 \leqslant p<+\infty)$ to the implicit parabolic differential equation

$$
f\left(x, t, u, D_{x} u, L u\right)=0,
$$

with the homogeneus Cauchy-Dirichlet conditions where $u=\left(u_{1}, u_{2}, \ldots, u_{h}\right)$, $D_{x} u=\left(D_{x} u_{1}, D_{x} u_{2}, \ldots, D_{x} u_{h}\right), L u=\left(\mathcal{L} u_{1}, \mathcal{L} u_{2}, \ldots, \mathcal{L} u_{h}\right)$.

\section{INTRODUCTION}

Let $Q_{T}=\Omega \times(0, T)$, where $\Omega$ is a bounded domain in $\mathbb{R}^{n}(n \geqslant 3)$ having the cone property and $T$ is a positive real number; let $f$ be a real-valued function defined in $Q_{T} \times \mathbb{R}^{h} \times \mathbb{R}^{n h} \times \mathbb{R}^{h}$; let $\mathcal{L}$ be a linear, second order, parabolic operator; let $Y$ be a nonempty, closed, connected and locally connected subset of $\mathbb{R}^{h}$. In the present paper we consider the implicit parabolic differential equation

$$
f\left(x, t, u, D_{x} u, L u\right)=0,
$$

where $u=\left(u_{1}, u_{2}, \ldots, u_{h}\right), \quad D_{x} u=\left(D_{x} u_{1}, D_{x} u_{2}, \ldots, D_{x} u_{h}\right)$, and $L u=$ $\left(\mathcal{L} u_{1}, \mathcal{L} u_{2}, \ldots, \mathcal{L} u_{h}\right)$.

We look for solutions $u \in W_{p}^{2,1}\left(Q_{T}, \mathbb{R}^{h}\right), n+2 \leqslant p<+\infty$, to equation (A) fulfilling the conditions

$$
\begin{gathered}
L u(x, t) \in Y \text { almost everywhere in } Q_{T}, \\
u(x, 0)=0 \text { in } \Omega \text { and } u(x, t)=0 \text { on } \partial \Omega \times(0, T) .
\end{gathered}
$$

If $h=1, Y=\mathbb{R}$ and, for every $(x, t, z, w) \in Q_{T} \times \mathbb{R} \times \mathbb{R}^{n}$, the equation $f(x, t, z, w, y)=0$ can be solved for $y,(\mathrm{~A})$ reduces to a quasi-linear equation

$$
\mathcal{L} u=\varphi\left(x, t, u, D_{x} u\right)
$$

Received 4 August 1994

Copyright Clearance Centre, Inc. Serial-fee code: 0004-9729/95 \$A2.00+0.00. 
and a variety of existence results, established in the framework of abstract evolution equations or resorting to techniques based on a priori bounds (see, for instance, respectively, $[1,4]$, and the references given there), are already available. Otherwise, as far as we know, nothing is known about the existence of solutions to the preceding problem.

The approach taken in this paper is similar to that previously introduced in [5] to study the solvability of the Dirichlet problem for an implicit elliptic differential equation. Namely, by using Theorem 1.1 of [7], we first reduce (A) - (B) to the parabolic differential inclusion

$$
L u(x, t) \in F\left(x, t, u, D_{x} u\right),
$$

where $F$ is a multiselection of the multifunction

$$
(x, t, z, w) \longrightarrow\{y \in Y: f(x, t, z, w, y)=0\}
$$

Next, through the same arguments employed to establish Theorem 3.1 in [5], we accomplish the proof of existence. This method produces rather general assumptions on the function $f$ and the set $Y$. For instance, we do not require that $f$ satisfy any Lipschitz condition for the last variable nor that the set $Y$ be compact. Moreover, "strongly" implicit problems can be solved by using the main result (Theorem 3.1) of this paper.

To give a more concrete idea of this, consider the following example (see Example 3.1). Let $g: Q_{T} \rightarrow \mathbb{R}$ be a measurable function satisfying

$$
\sup _{(x, t) \in Q_{T}}|g(x, t)|<2-(\pi / 2)
$$

and let $k: \mathbb{R} \rightarrow \mathbb{R}$ be the function defined by

$$
k(y)= \begin{cases}\frac{y-1}{|y-1|}\left(2+\sin \frac{3}{|y-1|}\right) \exp \left(\frac{-1}{|y-1|}\right) & \text { if } y \neq 1 \\ 0 & \text { if } y=1\end{cases}
$$

Then, for any $p \in[n+2,+\infty)$, there is a function $u \in W_{p}^{2,1}\left(Q_{T}\right)$ such that

$$
\begin{gathered}
k(\mathcal{L} u(x, t))=g(x, t)+\arctan u(x, t) \text { almost everywhere in } Q_{T}, \\
u(x, 0)=0 \text { for every } x \in \Omega \text { and } u(x, t)=0 \text { for every }(x, t) \in \Omega \times(0, T) .
\end{gathered}
$$

\section{Preliminaries}

Let $\Omega$ be a nonempty, bounded, open, connected subset of $\mathbb{R}^{n}, n \geqslant 3$, having the cone property; let $Q_{T}=\Omega \times(0, T)(T>0)$; let $p \in[n+2,+\infty)$. 
We denote by $W_{p}^{2,1}\left(Q_{T}\right)$ the space of all the functions $u \in L^{p}\left(Q_{T}\right)$ whose derivatives (in the distributional sense on $Q_{T}$ )

$$
\frac{\partial u}{\partial x_{i}}, \frac{\partial u}{\partial t}, \frac{\partial^{2} u}{\partial x_{i} \partial x_{j}} \quad(i, j=1, \ldots, n)
$$

belong to $L^{p}\left(Q_{T}\right)$. A norm on $W_{p}^{2,1}\left(Q_{T}\right)$ is introduced by defining

$$
\|u\|_{2,1, p}=\sum_{|\alpha| \leqslant 2}\left\|D_{x}^{\alpha} u\right\|_{p}+\left\|D_{t} u\right\|_{p}
$$

where $\alpha=\left(\alpha_{1}, \alpha_{2}, \ldots, \alpha_{n}\right),|\alpha|=\alpha_{1}+\alpha_{2}+\cdots+\alpha_{n}$ and

$$
D_{x}^{\alpha} u=\frac{\partial^{|\alpha|} u}{\partial x_{1}^{\alpha_{1}} \ldots \partial x_{n}^{\alpha_{n}}}, D_{t} u=\frac{\partial u}{\partial t} .
$$

The symbol $W\left(Q_{T}\right)$ stands for the space of all $u \in W_{p}^{2,1}\left(Q_{T}\right)$ such that $u(x, 0)=0$ for every $x \in \Omega$ and $u(x, t)=0$ for every $(x, t) \in \partial \Omega \times(0, T)$.

Given a positive integer $h$, we denote by $W_{p}^{2,1}\left(Q_{T}, \mathbb{R}^{h}\right)$ the space of all functions $u: Q_{T} \rightarrow \mathbb{R}^{h}, u=\left(u_{1}, u_{2}, \ldots, u_{h}\right)$, such that

$$
u_{i} \in W_{p}^{2,1}\left(Q_{T}\right) \text { for every } i=1,2, \ldots, h .
$$

The norm in this space is defined by

$$
|u|_{2,1, p}=\sum_{i=1}^{h}\left\|u_{i}\right\|_{2,1, p}, \quad u=\left(u_{1}, u_{2}, \ldots, u_{h}\right) \in W_{p}^{2,1}\left(Q_{T}, \mathbb{R}^{h}\right) .
$$

In a similar way one introduces the space $W\left(Q_{T}, \mathbb{R}^{h}\right)$. For every $u=$ $\left(u_{1}, u_{2}, \ldots, u_{h}\right) \in W\left(Q_{T}, \mathbb{R}^{h}\right)$, we set

$$
D_{x} u=\left(D_{x} u_{1}, D_{x} u_{2}, \ldots, D_{x} u_{h}\right),
$$

where

$$
D_{x} u_{i}=\left(\frac{\partial u_{i}}{\partial x_{1}}, \frac{\partial u_{i}}{\partial x_{2}}, \ldots, \frac{\partial u_{i}}{\partial x_{n}}\right)
$$

Finally, we write $|\cdot|$ (respectively $\|\cdot\|$ ) to denote the Euclidean norm of $\mathbb{R}^{h}$ (respectively, $\mathbb{R}^{n h}$ ); $d$ is the metric induced by $|\cdot|$ and 0 is the origin of $\mathbb{R}^{h}$.

The Sobolev Imbedding Theorem (see for instance [4, Lemma 3.3, p.80]) shows that, if $p \geqslant n+2$, then there is a constant $\eta$ such that

$$
|u(x, t)| \leqslant \eta|u|_{2,1, p}, \quad\left\|D_{x} u(x, t)\right\| \leqslant \eta|u|_{2,1, p}
$$

for every $(x, t) \in Q_{T}$ and every $u \in W\left(Q_{T}, \mathbb{R}^{h}\right)$.

Throughout this paper the letter

(*) $c$ denotes the smallest constant $\eta$ such that inequalities (1) hold for all the functions $u \in W\left(Q_{T}, \mathbb{R}^{h}\right)$. 
Let $\mathcal{L}$ be the linear, second order, parabolic differential operator defined by

$$
\mathcal{L} u=\frac{\partial u}{\partial t}-\sum_{i, j=1}^{n} a_{i j}(x, t) \frac{\partial^{2} u}{\partial x_{i} \partial x_{j}}+\sum_{i=1}^{n} a_{i}(x, t) \frac{\partial u}{\partial x_{i}}+a(x, t) u
$$

where $a_{i j}(i, j=1,2, \ldots, n)$ are real-valued continuous bounded functions defined in $Q_{T}, a_{i} \in L^{\sigma}\left(Q_{T}\right)$ with

$$
\sigma=\left\{\begin{array}{ll}
p & \text { if } p>n+2 \\
n+2+\epsilon & \text { if } p=n+2 \quad(\varepsilon>0)
\end{array} \quad(i=1,2, \ldots, n)\right.
$$

$a \in L^{p}\left(Q_{T}\right)$ and

$$
\sum_{i, j=1}^{n} a_{i j}(x, t) \xi_{i} \xi_{j} \geqslant \lambda \sum_{i=1}^{n} \xi_{i}^{2}
$$

for some $\lambda>0$, every $(x, t) \in Q_{T}$ and every $\xi_{1}, \xi_{2}, \ldots, \xi_{n} \in \mathbb{R}$. Theorem 9.1 of [4, p.341], ensures that $\mathcal{L}: W\left(Q_{T}\right) \longrightarrow L^{p}\left(Q_{T}\right)$ is a one-to-one, surjective, linear operator and that its inverse $\mathcal{L}^{-1}: L^{P}\left(Q_{T}\right) \longrightarrow W\left(Q_{T}\right)$ is continuous.

We define, for every $u=\left(u_{1}, u_{2}, \ldots, u_{h}\right) \in W\left(Q_{T}, \mathbb{R}^{h}\right)$,

$$
L u=\left(\mathcal{L} u_{1}, \mathcal{L} u_{2}, \ldots, \mathcal{L} u_{h}\right)
$$

It is immediately verified that $L$ is a linear one-to-one operator from $W\left(Q_{T}, \mathbb{R}^{h}\right)$ onto $L^{p}\left(Q_{T}, \mathbb{R}^{h}\right)$ and that the inverse operator $L^{-1}$ is continuous. We denote by $\left\|L^{-1}\right\|$ the norm of $L^{-1}$.

For definitions concerning multifunctions, the spaces $W^{i, p}\left(\Omega, \mathbb{R}^{h}\right)(i=1,2)$ and further details, see Section 2 of [5].

\section{EXISTENCE THEOREMS}

The main result of this paper is the following

THEOREM 3.1. Let $Y$ be a nonempty, closed, connected and locally connected subset of $\mathbb{R}^{h}$ and let $f$ be a real-valued function defined on $Q_{T} \times \mathbb{R}^{h} \times \mathbb{R}^{n h} \times Y$. Assume that:

( $\left.\mathbf{a}_{1}\right)$ For every $(x, t, z, w) \in Q_{T} \times \mathbb{R}^{h} \times \mathbb{R}^{n h}$ the function $y \rightarrow f(x, t, z, w, y)$ is continuous, $0 \in \operatorname{int}(f(x, t, z, w, Y))$ and the set $\{y \in Y: f(x, t, z, w, y)=$ 0\} has empty interior in $Y$.

(a. There is a set $\Lambda \subseteq Y \times Y, \Lambda$ dense in $Y \times Y$, such that for every $(x, t) \in$ $Q_{T}$ and $\left(y_{1}, y_{2}\right) \in \Lambda$ the set $\left\{(z, w) \in \mathbb{R}^{h} \times \mathbb{R}^{n h}: f\left(x, t, z, w, y_{1}\right)<0<\right.$ $\left.f\left(x, t, z, w, y_{2}\right)\right\}$ is open. 
(as) There is a countable set $\Lambda^{*} \subseteq Y \times Y, \Lambda^{*}$ dense in $Y \times Y$, so that for every $\left(y_{1}, y_{2}\right) \in \Lambda^{*}$ the set $\left\{(x, t, z, w) \in Q_{T} \times \mathbb{R}^{h} \times \mathbb{R}^{n h}: f\left(x, t, z, w, y_{1}\right)<\right.$ $\left.0<f\left(x, t, z, w, y_{2}\right)\right\}$ belongs to $\mathcal{L}\left(Q_{T}\right) \otimes \mathcal{B}\left(\mathbb{R}^{h} \times \mathbb{R}^{n h}\right)$.

$\left(a_{4}\right)$ There exist $r>0$ and $m \in L^{p}\left(Q_{T}\right)(n+2 \leqslant p<+\infty)$ such that $\left\|L^{-1}\right\|\|m\|_{p} \leqslant r$ and $\{y \in Y: f(x, t, z, w, y)=0, y$ is not a local extremum point for $f(x, t, z, w, \cdot)\} \subseteq B(0, m(x, t))$ for almost every $(x, t) \in Q_{T}$ and every $(z, w) \in \mathbb{R}^{h} \times \mathbb{R}^{n h}$ with $|z| \leqslant c r,\|w\| \leqslant c r$, where c satisfies $(*)$.

Then there is a function $u \in W\left(Q_{T}, \mathbb{R}^{h}\right)$ so that $L u(x, t) \in Y$ and $f(x, t, u(x, t)$, $\left.D_{x} u(x, t), L u(x, t)\right)=0$ for almost every $(x, t) \in Q_{T}$.

Proof: Fix $(x, t) \in Q_{T}$. For every $(z, w) \in \mathbb{R}^{h} \times \mathbb{R}^{n h}$, we define $F(x, t, z, w)=$ $\{y \in Y: f(x, t, z, w, y)=0, y$ is not a local extremum point for $f(x, t, z, w, \cdot)\}$.

It is a simple matter to see that all the assumptions of Theorem 1.1 in [7] are satisfied. Hence, the set $F(x, t, z, w)$ is nonempty and closed for any $(z, w) \in \mathbb{R}^{h} \times \mathbb{R}^{n h}$, and the multifunction $(z, w) \rightarrow F(x, t, z, w)$ is lower semicontinuous.

Let $A$ be a nonempty, connected, open subset of $Y$. If $(x, t, z, w) \in F^{-}(A)$ then there is $y \in A$ such that $f(x, t, z, w, y)=0$ and $y$ is not a local extremum point for $\xi \rightarrow f(x, t, z, w, \xi)$. By hypothesis $\left(a_{1}\right)$, this implies that there are two open sets $A_{1}, A_{2} \subseteq A$ so that $f\left(x, t, z, w, y_{1}\right)<0$ for every $y_{1} \in A_{1}$ and $f\left(x, t, z, w, y_{2}\right)>0$ for every $y_{2} \in A_{2}$. Bearing in mind that $\Lambda^{*} \cap\left(A_{1} \times A_{2}\right) \neq \emptyset$, we obtain $\left(y_{1}, y_{2}\right) \in$ $\Lambda^{*} \cap(A \times A)$ satisfying

$$
f\left(x, t, z, w, y_{1}\right)<0<f\left(x, t, z, w, y_{2}\right) .
$$

Conversely, let $(x, t, z, w) \in Q_{T} \times \mathbb{R}^{h} \times \mathbb{R}^{n h}$ and let $\left(y_{1}, y_{2}\right) \in \Lambda^{*} \cap(A \times A)$ be such that $f\left(x, t, z, w, y_{1}\right)<0<f\left(x, t, z, w, y_{2}\right)$. Since $A$ is connected, there is $y \in A$ so that $f(x, t, z, w, y)=0$. If $y$ is not a local extremum point for $\xi \rightarrow f(x, t, z, w, \xi)$, then $(x, t, z, w) \in F^{-}(A)$. Otherwise, assumption $\left(a_{1}\right)$ and Lemma 2.1 of [6] produce $y^{*} \in A$ such that $f\left(x, t, z, w, y^{*}\right)=0$ and $y^{*}$ is not a local extremum point for $\xi \rightarrow f(x, t, z, w, \xi)$. Therefore, in either case,

$$
\begin{aligned}
F^{-}(A)=\bigcup_{\left(y_{1}, y_{2}\right) \in \Lambda^{*} \cap(A \times A)}\left\{(x, t, z, w) \in Q_{T} \times \mathbb{R}^{h} \times \mathbb{R}^{n h}:\right. \\
\left.f\left(x, t, z, w, y_{1}\right)<0<f\left(x, t, z, w, y_{2}\right)\right\} .
\end{aligned}
$$

By hypothesis $\left(a_{3}\right)$, the preceding identity yields

$$
F^{-}(A) \in \mathcal{L}\left(Q_{T}\right) \otimes \mathcal{B}\left(\mathbb{R}^{h} \times \mathbb{R}^{n h}\right)
$$


Since the space $Y$ has a countable base of connected open sets, we see that the multifunction $F$ is $\mathcal{L}\left(Q_{T}\right) \otimes \mathcal{B}\left(\mathbb{R}^{h} \times \mathbb{R}^{n h}\right)$-measurable. Moreover, because of assumption $\left(a_{4}\right)$, for almost every $(x, t) \in Q_{T}$ one has

$$
\sup \{d(F(x, t, z, w), 0):|z| \leqslant c r,\|w\| \leqslant c r\} \leqslant m(x, t) .
$$

Now, we set $B_{r}=\left\{u \in W\left(Q_{T}, \mathbb{R}^{h}\right):|u|_{2,1, p} \leqslant r\right\}$ and $S_{r}=\overline{j\left(B_{r}\right)}$, where $j$ denotes the imbedding operator of $W_{p}^{2,1}\left(Q_{T}, \mathbb{R}^{h}\right)$ into $L^{p}\left((0, T) ; W^{1, p}\left(\Omega, \mathbb{R}^{h}\right)\right)$. The mapping $j$ is compact. In fact, let $\left\{u_{k}\right\}$ be a bounded sequence in $W_{p}^{2,1}\left(Q_{T}, \mathbb{R}^{h}\right)$; then $\left\{u_{k}\right\}$ is bounded in $L^{p}\left((0, T) ; W^{2, p}\left(\Omega, \mathbb{R}^{h}\right)\right)$ and $\left\{\frac{\theta u_{k}}{\partial t}\right\}$ is bounded in $L^{p}\left((0, T) ; L^{p}\left(\Omega, \mathbb{R}^{h}\right)\right)$. Since the imbedding of $W^{2, p}\left(\Omega, \mathbb{R}^{h}\right)$ into $W^{1, p}\left(\Omega, \mathbb{R}^{h}\right)$ is compact (see for instance [3, Theorem 11.2, p.31]), due to Theorem 1 of [2], there exists a subsequence of $\left\{u_{k}\right\}$ strongly converging in $L^{p}\left((0, T) ; W^{1, p}\left(\Omega, \mathbb{R}^{h}\right)\right)$.

Finally, by means of inequalities (1), for every $v \in S_{r}$ we obtain

$$
|v(x, t)| \leqslant c r \text { and }\left\|D_{x} v(x, t)\right\| \leqslant c r \text { almost everywhere in } Q_{T} .
$$

If $v \in S_{r}$, we define

$$
\begin{gathered}
G(v)=\left\{w \in L^{p}\left(Q_{T}, \mathbb{R}^{h}\right): w(x, t) \in F\left(x, t, v(x, t), D_{x} v(x, t)\right)\right. \\
\text { almost everywhere in } \left.Q_{T}\right\} .
\end{gathered}
$$

Owing to the properties of the multifunction $F$, the same arguments used in the proof of Theorem 3.1 in [5] can be applied to conclude that there is a continuous function

$$
g: S_{r} \longrightarrow L^{p}\left(Q_{T}, \mathbb{R}^{h}\right)
$$

satisfying $g(v) \in G(v)$ for all $v \in S_{r}$.

Obviously, the function $j \circ L^{-1} \circ g: S_{r} \rightarrow L^{p}\left((0, T) ; W^{1, p}\left(\Omega, \mathbb{R}^{h}\right)\right)$ is continuous. Moreover $j \circ L^{-1} \circ g\left(S_{r}\right) \subseteq S_{r}$, since for any $v \in S_{r}$ one has

$$
\left|L^{-1}(g(v))\right|_{2,1, p} \leqslant\left\|L^{-1}\right\|\|g(v)\|_{L^{p}\left(Q_{T}, \mathbb{L}^{h}\right)} \leqslant\left\|L^{-1}\right\|\|m\|_{p} \leqslant r
$$

because of (2) and (3). Thus, by the Schauder Fixed Point Theorem, there exists $v_{0} \in S_{r}$ such that $j \circ L^{-1} \circ g\left(v_{0}\right)=v_{0}$.

It is immediate to verify that the function $u=L^{-1}\left(g\left(v_{0}\right)\right)$ belongs to $W\left(Q_{T}, \mathbb{R}^{h}\right)$ and satisfies the conditions

$$
L u(x, t) \in F\left(x, t, u(x, t), D_{x} u(x, t)\right),|L u(x, t)| \leqslant m(x, t)
$$

almost everywhere in $Q_{T}$. This completes the proof. 
REMARK 3.1. It is of interest to note that the assumptions $\left(a_{2}\right)$ and $\left(a_{3}\right)$ of Theorem 3.1 are satisfied if, for example, the function $(x, t) \rightarrow f(x, t, z, w, y)$ is measurable for all $(z, w, y) \in \mathbb{R}^{h} \times \mathbb{R}^{n h} \times Y$ and the function $(z, w) \rightarrow f(x, t, z, w, y)$ is continuous for all $(x, t, y) \in Q_{T} \times Y$. Moreover, hypothesis $\left(a_{4}\right)$ is obviously satisfied whenever the set $Y$ is bounded.

The proof of Theorem 3.1 contains an existence result for parabolic differential inclusions, which is interesting enough to be stated separately.

THEOREM 3.2. Let $F: Q_{T} \times \mathbb{R}^{h} \times \mathbb{R}^{n h} \rightarrow 2^{\mathbb{R}^{h}}$ be a multifunction with nonempty closed values having the following properties:

$\left(\mathrm{h}_{1}\right)$ For almost every $(x, t) \in Q_{T}$, the multifunction $(z, w) \rightarrow F(x, t, z, w)$ is lower semicontinuous.

( $\left.\mathrm{h}_{2}\right) \quad$ The multifunction $F$ is $\mathcal{L}\left(Q_{T}\right) \otimes \mathcal{B}\left(\mathbb{R}^{h} \times \mathbb{R}^{\text {nh }}\right)$-measurable.

$\left(\mathrm{h}_{3}\right)$ There exist $r>0$ and $m \in L^{p}\left(Q_{T}\right)(n+2 \leqslant p<+\infty)$ such that $\left\|L^{-1}\right\|\|m\|_{p} \leqslant r$ and

$$
\sup \{d(F(x, t, z, w), 0):|z| \leqslant c r,\|w\| \leqslant c r\} \leqslant m(x, t)
$$

almost everywhere in $Q_{r}$, where $c$ satisfies (*).

Then there exists a function $u \in W\left(Q_{T}, \mathbb{R}^{h}\right)$ such that

$$
L u(x, t) \in F\left(x, t, u(x, t), D_{x} u(x, t)\right) \text { and }|L u(x, t)| \leqslant m(x, t)
$$

for almost every $(x, t) \in Q_{T}$.

A variety of special cases of Theorem 3.1 are particularly interesting. As an example, we present the following result, which makes more explicit the conclusion of Theorem 3.1.

THEOREM 3.3. Let $f_{1}, f_{2}, \ldots, f_{k}$ be $k$ real-valued functions defined in $\mathbb{R}^{h}$ and let $f$ be a continuous real-valued function defined in $Q_{T} \times \mathbb{R}^{h} \times \mathbb{R}^{n h} \times \mathbb{R}^{h}$. Let $Y$ be a nonempty, closed, connected and locally connected subset of $\bigcap_{i=1}^{k} f_{i}^{-1}(0)$ such that $0 \in \operatorname{int}(f(x, t, z, w, Y))$ and the set $\{y \in Y: f(x, t, z, w, y)=0\}$ has empty interior in $Y$ for all $(x, t, z, w) \in Q_{T} \times \mathbb{R}^{h} \times \mathbb{R}^{n h}$. Further, suppose that the hypothesis $\left(\mathrm{a}_{4}\right)$ of Theorem 3.1 holds. Then there is a function $u \in W\left(Q_{T}, \mathbb{R}^{h}\right)$ such that

$$
\begin{gathered}
f_{1}(L u(x, t))=0, f_{2}(L u(x, t))=0, \ldots, f_{k}(L u(x, t))=0 \\
f\left(x, t, u(x, t), D_{x} u(x, t), L u(x, t)\right)=0 \text { almost everywhere in } Q_{T} .
\end{gathered}
$$

Finally, to give a more precise idea of the possible applications of Theorem 3.1, we present the following example. 
EXAmple 3.1. Let $g: Q_{T} \rightarrow \mathbb{R}$ be a measurable function and let $k: \mathbb{R} \rightarrow \mathbb{R}$ be the function defined by

$$
k(y)= \begin{cases}\frac{y-1}{|y-1|}\left(2+\sin \frac{3}{|y-1|}\right) \exp \left(\frac{-1}{|y-1|}\right) & \text { if } y \neq 1 \\ 0 & \text { if } y=1\end{cases}
$$

Suppose

$$
\inf _{(x, t) \in Q_{T}} g(x, t)>-2+(\pi / 2) \text { and } \sup _{(x, t) \in Q_{T}} g(x, t)<2-(\pi / 2) .
$$

Then there is a function $u \in W\left(Q_{T}\right)$ such that

$$
k(\mathcal{L} u(x, t))=g(x, t)+\arctan u(x, t)
$$

almost everywhere in $Q_{T}$.

Proof: For every $(x, t, z, y) \in Q_{T} \times \mathbb{R} \times \mathbb{R}$, we put

$$
f(x, t, z, y)=k(y)-(g(x, t)+\arctan z) .
$$

It is easily proved that the function $f: Q_{T} \times \mathbb{R} \times \mathbb{R} \rightarrow \mathbb{R}$ so defined satisfies the assumptions $\left(a_{2}\right)$ and $\left(a_{3}\right)$ of Theorem 3.1. To verify hypothesis $\left(a_{1}\right)$, we observe that, for every fixed $(x, t, z) \in Q_{T} \times \mathbb{R}$, the function $y \rightarrow f(x, t, z, y)$ is continuous and the set $\{y \in \mathbb{R}: f(x, t, z, y)=0\}$ has empty interior. Moreover, since one has

$$
\lim _{k \rightarrow-\infty} k(y)=-2 \text { and } \lim _{k \rightarrow+\infty} k(y)=2,
$$

inequalities (4) yield $y_{1}, y_{2} \in \mathbb{R}$ satisfying $f\left(x, t, z, y_{1}\right)<0<f\left(x, t, z, y_{2}\right)$, so that $0 \in \operatorname{int}(f(x, t, z, \mathbb{R}))$. Finally, because of (4) and (5), there is $\rho>0$ such that

$$
f(x, t, z, y)<0 \text { for all }(x, t, z, y) \in Q_{T} \times \mathbb{R} \times(-\infty,-\rho)
$$

and

$$
f(x, t, z, y)>0 \text { for all }(x, t, z, y) \in Q_{T} \times \mathbf{R} \times(\rho,+\infty) .
$$

Therefore, assumption $\left(a_{4}\right)$ is satisfied too whenever we choose $m(x, t)=\rho,(x, t) \in$ $Q_{T}$, and $r \geqslant\left\|\mathcal{L}^{-1}\right\|\|m\|_{p}$. Now, Theorem 3.1 can be applied to get a function $u \in$ $W\left(Q_{T}\right)$ such that

$$
k(\mathcal{L} u(x, t))-(g(x, t)+\arctan u(x, t))=0
$$

for almost every $(x, t) \in Q_{T}$. 


\section{REFERENCES}

[1] H. Amann, 'Parabolic evolution equations and nonlinear boundary conditions', J. Differential Equations 72 (1988), 201-269.

[2] J.P. Aubin, 'Un théorème de compacité', C.R. Acad. Sci. Paris, Série I 256 (1963), 5042-5044.

[3] A. Friedman, Partial differential equations (Holt, Rinehart and Winston, New York, 1969).

[4] O.A. Ladyženskaja, V.A. Solonnikov and N.N. Ural'ceva, 'Linear and quasilinear equations of parabolic type', Transl. Math. Monographs 23 (American Mathematical Society, Providence, 1968).

[5] S.A. Marano, 'Implicit elliptic differential equations', Set-Valued Anal. 2 (1994), 545-558.

[6] B. Ricceri, 'Sur la semi-continuité inférieure des certaines multifonctions', C.R. Acad. Sci. Paris, Série I 294 (1982), 265-267.

[7] B. Ricceri, 'Applications de théorèmes de semi-continuité inférieure', C.R. Acad. Sci. Paris, Série I 285 (1982), 75-78.

Dipartimento di Matematica

Universita' di Catania

Viale A. Doria 6 - 95125 Catania

Italy 